\title{
Bridge between Abelian and Non-Abelian Fractional Quantum Hall States
}

\author{
N. Regnault ${ }^{1}$, M. O. Goerbig ${ }^{2}$, and Th. Jolicoeur ${ }^{3}$ \\ ${ }^{1}$ Laboratoire Pierre Aigrain, Département de Physique, \\ ENS, CNRS, 24 Rue Lhomond, F-75005 Paris, France \\ ${ }^{2}$ Laboratoire de Physique des Solides, CNRS UMR 8502, Univ. Paris-Sud, F-91405 Orsay cedex, France \\ ${ }^{3}$ Laboratoire de Physique Théorique et Modèles Statistiques, Univ. Paris-Sud, F-91405 Orsay cedex, France
}

\begin{abstract}
We propose a scheme to construct the most prominent Abelian and non-Abelian fractional quantum Hall states from $K$-component Halperin wave functions. In order to account for a onecomponent quantum Hall system, these $\mathrm{SU}(K)$ colors are distributed over all particles by an appropriate symmetrization. Numerical calculations corroborate the picture that $K$-component Halperin wave functions may be a common basis for both Abelian and non-Abelian trial wave functions in the study of one-component quantum Hall systems.

PACS numbers: 73.43.Nq, 71.10.Pm, 73.20.Qt
\end{abstract}

Trial wave functions play a central role in the study of the fractional quantum Hall effect (FQHE). Thus, the FQHE at filling factors $\nu \equiv n_{e l} / n_{B}=1 / 3,1 / 5, \ldots$, in terms of the electronic $n_{e l}$ and the flux $n_{B}=e B / h$ densities, respectively, has been explained with the help of Laughlin's wave function [1]. The states of the principle FQHE series $\nu=p /(2 s p \pm 1)$ have found a compelling interpretation in terms of composite-fermion $(\mathrm{CF})$ wave functions [2]. Other proposals include non-Abelian states, which may be described with the help of a Pfaffian wave function at half-filling [3] or a parafermionic generalization of it [4], at $\nu=K /(K+2)$, in terms of an integer $K$. All of these states may be viewed as sophisticated generalizations of Laughlin's wave function to describe incompressible quantum liquids.

In addition to these one-component wave functions, which treat fermions of only one type (spinless fermions), a two-component generalization has been proposed by Halperin to account for the spin degree of freedom [5]. A similar situation arises in bilayer quantum Hall systems where the bilayer index may be mimicked by an isospin, and the FQHE may equally be described in terms of twocomponent Halperin wave functions [6]. Multicomponent quantum Hall systems have recently attracted increasing interest because of the discovery of a particular quantum Hall effect in graphene, two-dimensional graphite 7]. Due to its fourfold spin-valley degeneracy, graphene in a strong magnetic field may indeed be viewed as a four-component quantum Hall system [8].

Motivated by graphene, as well as bilayer quantum Hall systems with non-polarized spin, two of us have recently generalized Halperin's wave function to the case with $K$ different components ("colors") [9]. Here, we show that these $\mathrm{SU}(K)$ Halperin wave functions may also play an important role in the understanding of the original one-component quantum Hall system, once symmetrized with respect to their color degree of freedom. This symmetrization of the $\mathrm{SU}(K)$ Halperin wave functions allows one to obtain the non-Abelian Read-Rezayi (RR) states at $\nu=K /(K+2)$ as well as its straight- forward generalization to $\nu=K /(n K+2)$ [4]. We corroborate this statement within exact-diagonalization (ED) studies on the sphere. Similarly, one may obtain the states at $\nu=K /(2 s K+1)$ of the $\mathrm{CF}$ series if one multiplies the $\mathrm{SU}(K)$ Halperin states by a product of $K(K-1) / 2$ permanents. Within Monte-Carlo calculations, we show that these states have a reasonably large overlap, for different accessible system sizes, with CF wave functions [2].

We consider the $K$-component Halperin wave function

$$
\Psi_{[m ; n]}^{(K)}=\prod_{k<l}\left(z_{k}-z_{l}\right)^{n} \prod_{i=1}^{K} \prod_{k_{i}<l_{i}}^{N / K}\left(z_{k_{i}}^{(i)}-z_{l_{i}}^{(i)}\right)^{m-n},
$$

where $z_{k_{i}}^{(i)}$ is the position of the $k_{i}$-th particle with color $(i)$ in the complex plane. Here, $N$ is the total number of particles, and the number of particles per color is, thus, $N^{(i)}=N / K$. The first Jastrow term is $\mathrm{SU}(K)$-symmetric and, thus, does not distinguish between different particle colors. Explicitly it may be written as $\prod_{k<l}\left(z_{k}-z_{l}\right)^{n} \equiv$ $\prod_{i<j} \prod_{k_{i}, k_{j}}\left(z_{k_{i}}^{(i)}-z_{k_{j}}^{(j)}\right)^{n} \prod_{i} \prod_{k_{i}<l_{i}}\left(z_{k_{i}}^{(i)}-z_{l_{i}}^{(i)}\right)^{n}$. Eq. (11), where we have omitted an overall Gaussian factor, represents a particular form of $\mathrm{SU}(K)$ Halperin wavefunctions [9] where all intra-color correlations are the same, with an exponent $m$, and all inter-color correlations are described by the exponent $n$. In this form, it describes FQHE states at a total filling factor 10]

$$
\nu=\frac{K}{n K+(m-n)} \quad \text { with } \quad \delta_{[m ; n]}=m,
$$

where $\delta_{[m ; n]}$ denotes the shift in the sphere geometry used in our ED calculations.

One notices that this state corresponds to a state of high symmetry because the different color groups are equally occupied, i.e. the generalized polarizations of the $\mathrm{SU}(K)$ spin are all zero. These polarizations are associated with the elements of the Cartan algebra, the set 
of the $K-1$ generators of $\mathrm{SU}(K)$ that mutually commute. The state (1) is, therefore, invariant with respect to color permutations [Weyl group of $\mathrm{SU}(K)$ ]. We implement this symmetry in the ED studies and use appropriate Haldane pseudopotentials [11] to generate the $\mathrm{SU}(K)$ Halperin state, as described in Ref. 9].

- Non-Abelian states. A compelling approach to construct the bosonic non-Abelian RR states at $\nu=K / 2$ has been provided by Cappelli et al [12]. They may indeed be obtained from the generalized Halperin wave function $\Psi_{[2 ; 0]}^{(K)}, \Psi_{R R}^{(K)}=\mathcal{S} \Psi_{[2 ; 0]}^{(K)}$, where the $\mathcal{S}$ symbol stands for the symmetrization over all possibilities to associate $K$ colors to all $N$ particles. A straightforward generalization to fermions is obtained with the help of the $\Psi_{[3 ; 1]}^{(K)}$ state,

$$
\Psi_{R R}^{(K)}=\mathcal{S}^{\prime} \Psi_{[3 ; 1]}^{(K)},
$$

where $\mathcal{S}^{\prime}$ has a similar meaning as $\mathcal{S}$, except that it removes those cases, which are equivalent up to global color permutation in order to avoid accidental cancelation due to the inherent fermionic nature of the $[3 ; 1]$ state. Notice that $\mathcal{S}^{\prime}$ may also be used for a bosonic state if one changes its normalization. Very recently, the states (3) and their degeneracy have been studied on the torus [13].

The symmetrization procedure may be applied directly to any $N$-body $\mathrm{SU}(K)$ Fock state. It consists of discarding any component of this state, which has at least one orbital with an occupancy greater than one, and of summing all components with the same orbital occupancy regardless of their color pattern. Furthermore, if we consider linear combinations of $N$-body states which are invariant under the discrete symmetries of the Weyl group, only the maximally symmetric ones survive this symmetrization procedure.

In order to test the above construction, we have checked by ED that one reproduces, up to machine precision, the RR states at $\nu=K /(K+2)$ from their Halperin counterparts for $K=2$ (for $N=4,6, \ldots 12$ particles) at $\nu=1 / 2$ (Pfaffian state), $K=3(N=6,9$, and 12) at $\nu=3 / 5$, and $K=4(N=4$ and $N=8)$ at $\nu=2 / 3$. We emphasize the large Hilbert space dimension due to the internal degree of freedom; e.g., for $K=3$ and $N=12$, the dimension of the largest subspace is 212121434 if one accounts for all discrete Weyl symmetries of $S U(3)$. Thus, this method to generate RR states is more involved as compared to using an appropriate $(K+1)$-body interaction (see e.g. 14]) or the "squeezing" technique [15].

Beyond these ground-state properties, Capelli et al. conjectured that the RR quasihole states may be obtained from the corresponding Halperin wave functions [12]. We have checked this hypothesis by ED for $K=2$, 3 , and 4 in the case of the $[3 ; 1]$ state. If one adds one flux quantum to the RR state, one generates $K$ quasiholes of a particular degeneracy [16], which may be sorted with respect to the eigenvalues of the angular momentum oper-

\section{$\mathbf{N}=\mathbf{8}, \mathbf{K}=\mathbf{2}$ : $\quad 1111000000001111$ \\ $\mathbf{N = 6 , K = 3 ~ : ~} \quad 11000011000011$ \\ $\mathbf{N = 9 , K = 3 ~ : ~} 111000000111000000111$}

FIG. 1: Thin-torus limit of $\mathcal{S}^{\prime} \Psi_{[1 ; 3]}^{(K)}$ for different values of $K$ and $N$. The particles phase-separate into $K$ repelling droplets, as expected for $m<n$. In general, these highest weights and the "squeezing" technique [15] do neither lead to a unique quantum state nor a state identical to that obtained by color symmetrization.

ators $L^{2}$ and $L_{z}$. In contrast to these states, the addition of one flux quantum to the $\mathrm{SU}(K)$ Halperin wave function $[3 ; 1]$ generates a larger set of degenerate quasiholes. However, we find that the degeneracy counting with respect to $L^{2}$ and $L_{z}$ is the same if we limit ourselves to the maximally symmetric sector of the Weyl group.

Notice that if both the Halperin and the RR states had the same degeneracy for any number, larger than one, of added flux quanta, this would preclude non-Abelian quasiparticle statistics because of the inherent Abelian nature of the excitations of the Halperin state. This is, however, not the case - the degeneracies are different for two added flux quanta. The symmetrization, thus, removes all unwanted states in the $\mathrm{SU}(K)$ case, and one obtains the correct degeneracy of the RR quasihole states, in agreement with their non-Abelian character. For a complete understanding of quasihole excitations in the framework of Halperin wave functions, additional conformal-field theoretical (CFT) studies would be required, which are beyond the scope of the present paper.

Beyond the $K$-color $[3 ; 1]$ states, which reproduce to great numerical accuracy the RR states at $\nu=K /(K+$ $2)$, one may investigate a generic $[m ; n]$ state. Consistency of the symmetrization procedure requires $m$ and $n$ to be both odd (even) in a fermionic (bosonic) onecomponent state. The $1 / \mathrm{m}$ Laughlin state is reproduced for $m=n$. For $m<n$, the $[m ; n]$ state is unstable in the generalized 2D plasma picture, and the (classical) particles of different colors undergo a phase-separate [10]. However, the quantum state $\mathcal{S}^{\prime} \Psi_{[m ; n]}^{(K)}$ is, in view of the analyticity condition for the lowest Landau level (LL), a valid candidate and one may look at the thin torus limit i.e. the highest weight as defined in 15]. In this limit, we clearly see the analogue of the plasma instability (Fig. 1): the $N$ particles tend to form $K$ mutually repelling clusters of $N / K$ particles. Moreover, even if $\mathcal{S}^{\prime} \Psi_{[1 ; 3]}^{(K)}$ has the same filling factor and shift as the non-Abelian states with reverse flux attachment [17], the overlap is low ( 0.659 for $K=3$ and $N=6$ ). Therefore, such states may not be related to homogeneous FQHE states.

The $m>n$ case is stable with respect to phase separation [10] and is, thus, a promising candidate for a homogeneous state. If we choose $m-n=2$, which also 


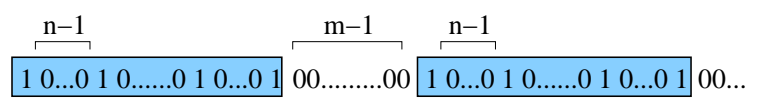

FIG. 2: Thin-torus limit of $\mathcal{S}^{\prime} \Psi_{[m ; n]}^{(K)}$. The shaded boxes indicate clusters of $K$ particles. The spacing between the clusters is determined by the exponent $m$, whereas that within each cluster by $n$, i.e. the inter-color correlations. $n=0$ (for bosons) corresponds to particles on the same site within each cluster.

accounts for the above-mentioned case $m=3, n=1$, we obtain generalized RR states [4] at filling factors $\nu=K /(K n+2)$. One may, therefore, conjecture that for every pair $[m ; n](m>n)$ with the same parity, the trial wave function $\mathcal{S}^{\prime} \Psi_{[m ; n]}^{(K)}$ yields a series of states at filling factors $\nu=K /(K n+r)$, the excitations of which may obey non-Abelian statistics ( $n$ even or odd for bosons or fermions, respectively). Here, we have defined $r=m-n$. Fig. 2 shows the generic highest weight limit of the states $\mathcal{S}^{\prime} \Psi_{[m ; n]}^{(K)}$ which we have obtained by ED for different values of $m, n, K$, and $N$. We have checked numerically that the application of the "squeezing" technique [15] applied to this highest weight for $m=5, n=1(K=2)$ yields the same quantum state as $\mathcal{S}^{\prime} \Psi_{[5 ; 1]}^{(2)}$. More generally, squeezing spans a subspace which $\mathcal{S}^{\prime} \Psi_{[5 ; 1]}^{(K)}$ belongs to. The pattern consists of $N / K$ equally spaced clusters with $K$ particles per cluster. Whereas the separation between the particles within each cluster is $n-1$ (the bosonic $n=0$ represents a cluster where all $K$ particles are on the same site), the inter-cluster separation is governed by the intra-color correlation exponent $m$. Before symmetrization, the particles per cluster may be viewed as $K$ particles of different color. This must be contrasted to the unstable case $m<n$, discussed above, where $N / K$ particles of the same color clusterize into $K$ repelling droplets. This indicates a certain duality, where the unstable Halperin states may be viewed as (stable) Halperin states of $N / K$ pseudo-colors with $K$ "particles" per color.

In order to obtain insight into the statistical properties of the quasihole excitations of the $[5 ; 1]$ states, we have investigated their degeneracies when adding one or more flux quanta (for $K=2$ and 3 ). Above a certain number of added flux quanta, the degeneracies of the states $\mathcal{S}^{\prime} \Psi_{[5 ; 1]}^{(K)}$ are indeed different from $\Psi_{[5 ; 1]}^{(K)}$ restricted to the maximally symmetric sector. Without being a strong proof, these results indicate that the quasihole excitations may be non-Abelian, as for the RR states.

Very recently, Bernevig and Haldane [18] have proposed a similar series for bosonic states at $\nu=K / r$, using Jack polynomials. This series matches ours for $n=0$, and both types of states have the same shift on the sphere $(\delta=r)$. We have checked numerically that both states have the same thin-torus limit. However, in our case, $r$ must be even and we recover only half of their states.
- States of the CF series. When $m$ and $n$ have different parity, the symmetrization procedure fails in generating fermionic or bosonic states. This problem may be fixed by multiplying the $\Psi_{[m ; n]}^{(K)}$ state with the correct object which restores the right statistics for the final onecomponent wave function. To illustrate this approach, we first consider a modified $K=2[3 ; 2]$ Halperin wave function at $\nu=2 / 5$,

$$
\tilde{\Psi}_{[3,2]}^{(2)}=\operatorname{perm}\left[M^{(1,2)}\right] \Psi_{[3,2]}^{(2)}\left(z_{1}^{(1)}, \ldots, z_{N}^{(1)}, z_{1}^{(2)}, \ldots, z_{N}^{(2)}\right),
$$

in terms of the $N \times N$ matrix

$$
M_{k_{i}, k_{j}}^{(i, j)}=\left[z_{k_{i}}^{(i)}-z_{k_{j}}^{(j)}\right]^{-1}
$$

$i, j=1,2$ here, and perm $\left[M^{(1,2)}\right]=\sum_{\{\sigma\}} \prod_{k=1}^{N} M_{k, \sigma(k)}^{(1,2)}$ is the permanent of $M^{(1,2)}$, where the sum is over all $\sigma$ permutations of $N$ elements. $\mathcal{S}^{\prime} \tilde{\Psi}_{[3 ; 2]}^{(2)}$ corresponds to a fermionic two-component state. The symmetrized wave function (44), as a candidate for the FQHE at $\nu=2 / 5$ has been studied by Yoshioka et al., and one obtains a large overlap with the ED ground state for a Coulomb interaction [19]. This wave function, also called "Gaffnian", has recently been studied within CFT and may support non-Abelian quasi-particle excitations [20].

To generalize this construction to higher values of $K$, we propose the following wave function

$$
\tilde{\Psi}_{[3,2]}^{(K)}=\prod_{i<j} \operatorname{perm}\left[M^{(i, j)}\right] \Psi_{[3 ; 2]}^{(K)},
$$

in terms of a product of $K(K-1) / 2$ permanents of the matrix (5). These wave functions may describe states at filling factors $\nu=K /(2 K+1)$, which corresponds to the principle series of two-flux $\mathrm{CF}$ 2]. Notice further that both $\mathcal{S}^{\prime} \tilde{\Psi}_{[3 ; 2]}^{(K)}$ and Jain's CF wave function are found at the same shift $\delta=K+2$ on the sphere. The utility of the wave function (6) as an alternative to Jain's CF construction was already pointed out by Morf in 2000 21]. Moreover, the wave function (6) is similar, but not equivalent to a generalization of the Gaffnian [20]. The function (6) may be related to $r=K+1$ and the highest weights as described in Fig. 2 (see [18] for $K=2$ ). However, it has been shown that the CFTs associated with Gaffnian-type functions are non-unitary and may, thus, yield to critical states with vanishing gap [20, 22].

By Monte-Carlo integration, we have studied quantitatively the overlap between $\mathcal{S}^{\prime} \tilde{\Psi}_{[3 ; 2]}^{(K)}$ and the CF wave function $\Psi_{C F}^{(K)}$ for $K$ filled pseudo-LLs [23], for $K=2$ $(\nu=2 / 5)$ and $K=3(\nu=3 / 7)$ and various values of $N$. The results are displayed in Tab. [) The overlaps for all studied system sizes are above $95 \%$. The strong statement that both approaches are equivalent, as indicated by the large overlap of the different trial wave 


\begin{tabular}{c|c|c|c|c|c}
$N$ & 6 & 8 & 10 & 12 & 14 \\
\hline $\mathcal{O}_{\nu=2 / 5}$ & $0.991(3)$ & $0.982(4)$ & $0.977(5)$ & $0.972(5)$ & $0.968(6)$ \\
\hline \hline$N$ & 6 & 9 & 12 & 15 & \\
\hline $\mathcal{O}_{\nu=3 / 7}$ & $0.993(3)$ & $0.979(4)$ & $0.963(5)$ & $0.954(7)$ &
\end{tabular}

TABLE I: Overlap between the CF wave functions $\Psi_{C F}^{(K)}$ with $K$ filled pseudo-LLs and $\mathcal{S}^{\prime} \tilde{\Psi}_{[3 ; 2]}^{(K)}$ for $K=2$ (at $\nu=2 / 5$, upper row) and $K=3$ (at $\nu=3 / 7$, lower row). $\mathcal{O}_{\nu}=\left|\left\langle\Psi_{C F}^{(K)} \mid \mathcal{S}^{\prime} \tilde{\Psi}_{[3 ; 2]}^{(K)}\right\rangle\right|$ is computed using Monte-Carlo integration with $10^{6}$ iterations. The numerical error on the last digit is indicated in parenthesis.

functions, would, however, require a detailed comparison of the quasiparticle excitations and their statistical properties, which is beyond the scope of this paper. Nevertheless, we stress that the generic $2 s$ flux $\mathrm{CF}$ also fits within the multicomponent scheme if we choose $n=2 s$ and $m-n=1$.

In order to obtain a better understanding of the relation between $\mathrm{CF}$ trial wave functions and $\mathcal{S}^{\prime} \tilde{\Psi}_{[2 s+1 ; 2 s]}^{(K)}$, we rewrite the $\mathrm{SU}(K)$ Halperin wave function as a product of two others, $\Psi_{[2 s+1 ; 2 s]}^{(K)}=\Psi_{[2 s ; 2 s]}^{(K)} \Psi_{[1 ; 0]}^{(K)}$. The SU $(K)$ symmetric one, $\Psi_{[2 s ; 2 s]}^{(K)}$, mimics the attachment of $2 s$ flux quanta per particle of any color and is, thus, common to both approaches, and $\Psi_{[1 ; 0]}^{(K)}$ is a product of $K$ Slater determinants for each color, i.e. the particles of each color form a liquid at an effective color filling $\nu_{i}^{*}=1$ with no correlations between different colors. Beyond this similarity with $\mathrm{CF}$ wave functions, we emphasize the following difference: in the $\mathrm{CF}$ approach, one describes particles of only one color which fill $K$ pseudo-LLs. This generates non-analytic components in the wave function, which must thus be projected to the lowest LL [2]. In our case, the trial wave function is naturally in the lowest LL, but the price to pay is the introduction of $K$ artificial colors, accompanied by the symmetrization procedure. Furthermore, in contrast to Jain's wave functions and the above non-Abelian states, one needs to introduce the permanent in order to describe particles with well-defined statistical properties.

We have, furthermore, investigated states with $m-n=$ -1 , the filling factor and shift on the sphere of which match those of their $\mathrm{CF}$ analogues with reversed flux attachment. However, the overlap with the $\mathrm{CF}$ wave function at $\nu=2 / 3$ drops from $0.901(3)$ for $N=6$ down to 0.097(2) for $N=12$. This corroborates the argument to discard unstable Halperin wave functions, as for the description of non-Abelian states.

In conclusion, we have shown that $K$-component Halperin wave functions $\Psi_{[m ; n]}^{(K)}$, with $m>n$ yield a common basis for the description of one-component FQHE states. The color symmetrization of states with $m$ and $n$ of the same parity ( $m, n$ odd for fermions and $m, n$ even for bosons) yields the generalized non-Abelian RR states for $m-n=2$. If $m$ and $n$ have different parity and if the Halperin wave function $\Psi_{[m ; n]}^{(K)}$ is multiplied by a product of $K(K-1) / 2$ permanents with respect to the different colors, the symmetrization yields wave functions of high overlap with Jain's CF states at $\nu=K /(n K+1)$, for even $n$ and $m-n=1$. The colors correspond to pseudoLLs in the CF picture, but the wave functions $\Psi_{[m ; n]}^{(K)}$ are fully in the lowest LL and need, thus, not be projected. A natural question arises whether a generalization to odd values of $r=m-n \neq 1$ may describe further CF-type states at $\nu=K /(n K+r)$ [24].

We acknowledge fruitful discussions with A. Bernevig, R. Santachiara, and J. Slingerland. We thank R. Morf for communicating us his talk at the 2001 EPS conference in Montreux and S. Simon for pointing out the relation of Eqs. (44) and (6) with the generalized Gaffnian. This work was funded by the Agence Nationale de la Recherche under Grant No. ANR-07-JCJC-0003-01.

[1] R. B. Laughlin, Phys. Rev. Lett. 50, 1395 (1983).

[2] J.K. Jain, Phys. Rev. Lett. 63, 199 (1989).

[3] G. Moore and N. Read, Nucl. Phys. B 360, 362 (1991).

[4] N. Read and E. Rezayi, Phys. Rev. B 59, 8084 (1999).

[5] B. I. Halperin, Helv. Phys. Acta 56, 75 (1983).

[6] For a review, see Perspectives in Quantum Hall Effects, edited by S. Das Sarma and A. Pinczuk (John Wiley, New York, 1997).

[7] K. S. Novoselov, A. K. Geim, S. V. Morosov, D. Jiang, M. I. Katsnelson, I. V. Grigorieva, S. V. Dubonos, and A. A. Firsov, Nature 438, 197 (2005); Y. Zhang, Y.-W. Tan, H. L. Stormer, and P. Kim, ibid. 201.

[8] A. H. Castro Neto, F. Guinea, N. M. R. Peres, K. S. Novoselov, and A. K. Geim, arXiv:0709.1163.

[9] M. O. Goerbig, and N. Regnault, Phys. Rev. B 75, 241405(R) (2007).

[10] R. de Gail, N. Regnault, and M. O. Goerbig, Phys. Rev. B 77, 165310 (2008).

[11] F. D. M. Haldane, Phys. Rev. Lett. 51, 605 (1983).

[12] A. Cappelli, L. S. Georgiev and I. T. Todorov, Nucl. Phys. B 599, 499 (2001).

[13] E. Ardonne, E. J. Bergholtz, J. Kailasvuori, and E. Wikberg, J. Stat. Mech. P04016 (2008).

[14] S.H. Simon, E.H. Rezayi, N.R. Cooper, Phys. Rev. B 75, 195306 (2007).

[15] B. A. Bernevig and F. D. M. Haldane, arXiv:0707.3637.

[16] E. Ardonne, J. Phys. A 35447 (2002); N. Read, Phys. Rev. B 73, 245334 (2006).

[17] Th. Jolicoeur, Phys. Rev. Lett. 99, 036805 (2007).

[18] B. A. Bernevig and F. D. M. Haldane, arXiv:0803.2882.

[19] D. Yoshioka, A. H. MacDonald, and S. M. Girvin, Phys. Rev. B 38, 3636 (1988).

[20] S. H. Simon, E. H. Rezayi, N. R. Cooper, and I. Berdnikov, Phys. Rev. B 75, 075317 (2007).

[21] R. Morf, talk at the European Physical Society Conference of the Condensed Matter Division (EPS-CMD18), Montreux 2000.

[22] N. Read, arXiv:0711.0543. 
[23] J.K. Jain and R.K. Kamilla, Int. J. Mod. Phys. B 11, 2621 (1997); Phys. Rev. B 55, R4895 (1997).
[24] see also E. Ardonne and J. Slingerland, in preparation. 